\title{
Splicing of the cholesterol homeostasis protein LXR is a prognostic indicator for triple negative breast cancer patients
}

\author{
P. Lianto ${ }^{1}$, T.A. Hughes ${ }^{2}$, B.J. Moore ${ }^{1}$ and L.J. Thorne ${ }^{1}$ \\ ${ }^{1}$ School of Food Science and Nutrition, University of Leeds, Leeds, LS2 9JT, UK and \\ ${ }^{2}$ School of Medicine, University of Leeds, Leeds, LS9 7TF, UK
}

There are positive correlations between hypercholesterolmia and BCa risk. LXR $\alpha / \mathrm{NR} 1 \mathrm{H} 3$ and LXR $\beta / \mathrm{NR} 1 \mathrm{H} 2$, known as regulators of cholesterol metabolism, are often over-expressed in breast cancer (BCa). LXRs mechanistically link cholesterol metabolism to BCa progression by reacting to side-chain hydroxylated cholesterol moiesties (scOHC; cholesterol metabolite) via ligand binding domains (LBD) and bind the genome via DNA binding domains (DBD $)^{(1,2)}$. Multiple splice variants of LXR $\alpha$ and LXR $\beta$ with alterations in the LBD, the DBD, and/or the Activation Function (AF) domain have been reported ${ }^{(3)}$. LXR splice variants annotated in NCBI, ENSEMBL and UNIPROT databases show divergence in domains critical for co-factor recruitment. We have previously shown co-factors are critical for distinguishing LXR function between oestrogen receptor positive (ER+) and negative (ER-) BCa subtypes ${ }^{(4)}$. As the expression and function of LXR splice variants in BCa have not been investigated before, we set out to evaluate the pattern and clinical significance of LXR splicing in BCa.

RT-qPCR and immunoblotting were performed to ascertain the range of splice variants expressed in a panel of BCa cell lines (ER+: MCF7, BT474; ER-: MDA.MB.468, MDA.MB.231, MDA.MB.157; Her2+: MDA.MB.453) and whether isoform abundance changed in response to synthetic (GW3965) and endogenous (26-hydroxycholesterol [26-OHC]) LXR ligands; changes in expression were determined with one-way ANOVA with Holm-Sidak correction. LXR isoforms were identified and validated in cell lines and were then quantified in tumours from 32 patients with triple negative breast cancer (TNBC) (15/HY/0025); associations with disease-free survival (DFS) were calculated using log-rank Mantel-Cox test.

Relative to a liver cell line (HEPG2 cells), LXR $\beta$ was expressed at a higher level in BCa lines than LXR $\alpha$. Multiple LXR $\alpha$ splice variants were present at mRNA and protein level, whilst just the LXR $\beta 1$ splice variant detectable by immunoblotting. Full length $\mathrm{LXR} \alpha 1$ was the predominant LXR $\alpha$ isoform in liver and TNBC cells and LXR $\alpha$ 4, which lacks a DBD and has a truncated AF, was the predominant isoform in ER+ cells. LXR $\alpha 1$ was inducible by GW3965 in all cell lines tested (p<0.05). LXR $\alpha x 4$ was dectable and inducible in MCF7 and MDA.MB.453 cells $(p<0.01)$. In ER-patients, high tumour levels of LXR $\alpha 1$ was associated with worse DFS $(p=0.0023)$. High levels of LXR $\alpha 4(p=0.026)$ and high LXR $\beta(p=0.049)$ was associated with longer DFS.

These data show fully functional LXR $\alpha$ is associated with worse survival. In contrast, patients with tumours having high expression of truncated LXR $\alpha$ or high LXR $\beta$, had longer DFS. Understanding the differences between gene targets of full length LXR $\alpha$, its splice variants, and LXR $\beta$ in BCa will help establish if the associations we idenitified here are causal. LXR splice analysis may improve prognostic accuracy, and identify patients for whom cholesterol lowering may improve survival.

1. Nelson ER, Wardell SE, Jasper JS, et al. (2013) Science 342, 1094-1098.

2. Segala G, David M, de Medina P, et al. (2017) Nat Commun 8, 1903.

3. Annalora AJ, Marcus CB, \& Iversen PL (2020). Drug Metab Dispos 48, 272-287.

4. Hutchinson SA, Lianto P, Roberg-Larsen H, et al. (2019). Nutrients 11, 2618. 